\title{
Optimal and real-time control potential of a diesel-electric powertrain
}

\author{
Martin Sivertsson* Lars Eriksson* \\ * Vehicular Systems, Dept. of Electrical Engineering, Linköping \\ University, SE-58183 Linköping, Sweden, \{marsi\}@isy.liu.se.
}

\begin{abstract}
Real-time control strategies and their performance related to the optimal control trajectories for a diesel-electric powertrain in transient operation are studied. The considered transients are steps from idle to target power. A non-linear four state-three input mean value engine model, incorporating the important turbocharger dynamics, is used for this study. The strategies are implemented using the SAE J1939-standard for engine control and evaluated compared to both the optimal solution and the solution when the engine is restricted to follow its stationary optimal line. It is shown that with the control parameters tuned for a specific criteria both engine control strategies in the SAE J1939-standard, speed control and load control, can achieve almost optimal results, where engine load controlled shows a better trade-off between fuel economy and duration. The controllers are then extended and it is shown that it is possible to control the powertrain in a close to optimal way using the SAE J1939-standard, both with the engine speed and load controlled. However the mode where the engine is load controlled is seen to be more robust.
\end{abstract}

\section{INTRODUCTION}

The diesel-electric powertrain, such as the BAE Systems Torq $\mathrm{E}^{\mathrm{TM}}$, see Fig. 1, offers the potential to increase the performance and lower the fuel consumption, since it decouples the diesel engine from the wheels. Through this electrification of the powertrain the engine speed can be chosen freely which also enables the powertrain to produce maximum power from standstill. This in combination with the torque characteristics of the electric motors can thus increase performance and potentially lower the fuel consumption.

During stationary operation the desired operating point can be found from the combined efficiency map of the engine-generator (GENSET). An open question is how to optimally control the GENSET between two different outputs, especially when the diesel engine is turbocharged. In transient operation the turbocharger dynamics limit the changes in load and speed that can be achieved, often referred to as turbocharger lag, see Rakopoulos and Giakoumis [2009]. The absence of an energy storage also makes the system more restricted and difficult to manage, compared to a series hybrid, that can use the energy storage to compensate for the dynamics of the engine, since all power consumed has to be produced by the GENSET. Therefore efficient transient control is of high importance, any delay in power response of the GENSET will also result in delay in power at the wheels.

Previous papers have studied how to best exploit the extra degree of freedom available in a diesel-electric and optimally control the engine-generator (GENSET) from idle to target power and energy, see Sivertsson and Eriksson $[2013 \mathrm{a}, \mathrm{b}]$. The main contribution of this paper is the study of the potential performance of different control strategies using the control principles used in industry, i.e. the SAE
J1939-standard for engine control, see SAE J1939 Standard [2013]. Two main approaches are discussed and implemented with the control parameters tuned for minimum fuel or minimum time. This is then performed for several cases and the results are related to the previous optimal results, investigating the potential for optimal control. As a further contribution the controllers are extended and it is shown that it is possible to control the GENSET in an optimal manner using the SAE J1939-standard.

The literature regarding diesel-electric powertrains is rather scarce. For series hybrids on the other hand, where the GENSET is augmented with an energy storage, there are several publications. A common approach is to use the stationary map to generate setpoints for the GENSET, see Yoo et al. [2009], Cairano et al. [2012], Sezer et al. [2011]. This optimization does not consider the transient effects of the GENSET and therefore raises the question if the optimal setpoint actually is the operating point with highest efficiency. Another approach is to limit the change in requested power from the GENSET so the controller can maintain the GENSET operating close to its stationary optimal line, see Cairano et al. [2012], Yoo et al. [2009]. This means that the energy storage needs provide a larger part of the requested power, but it also assumes that it is optimal to follow the stationary optimal line in transients. Whether these assumptions are true or not for turbocharged GENSETs is also studied in this paper.

\section{MODEL}

The modeled powertrain consists of a 6-cylinder 12.7-liter SCANIA diesel engine with a fixed-geometry turbine and a wastegate for boost control, equipped with a generator. The model is a nonlinear, four state, three input mean value engine model (MVEM), used together with models for the generator losses. The diesel engine model 


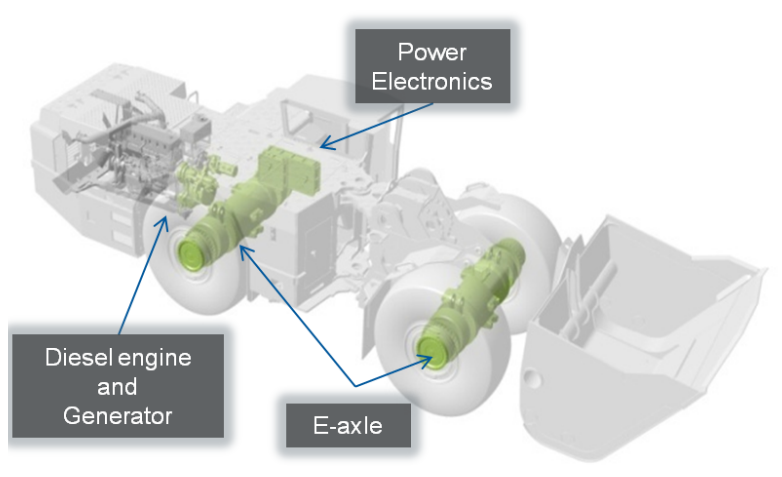

Fig. 1. BAE Systems Torq $E^{\mathrm{TM}}$ powertrain.

is the same as the one used in Sivertsson and Eriksson [2012], augmented with a model for the generator losses as in Sivertsson and Eriksson [2013a].

The states of the MVEM are engine speed, $\omega_{i c e}$, inlet manifold pressure, $p_{i m}$, exhaust manifold pressure, $p_{e m}$, turbocharger speed, $\omega_{t c}$. The controls are injected fuel mass, $u_{f}$, wastegate position, $u_{w g}$, and generator power, $P_{g e n}$. The engine model consists of two control volumes, intake and exhaust manifold, and four restrictions, compressor, engine, turbine, and wastegate. The control volumes are modeled with the standard isothermal model, using the ideal gas law and mass conservation. The engine and turbocharger speeds are modeled using Newton's second law. The governing differential equations of the MVEM are:

$$
\begin{aligned}
\frac{d \omega_{i c e}}{d t} & =\frac{1}{J_{g e n s e t}}\left(T_{i c e}-\frac{P_{\text {mech }}}{\omega_{i c e}}\right) \\
\frac{d p_{i m}}{d t} & =\frac{R_{a} T_{i m}}{V_{i m}}\left(\dot{m}_{c}-\dot{m}_{a c}\right) \\
\frac{d p_{e m}}{d t} & =\frac{R_{e} T_{e m}}{V_{e m}}\left(\dot{m}_{a c}+\dot{m}_{f}-\dot{m}_{t}-\dot{m}_{w g}\right) \\
\frac{d \omega_{t c}}{d t} & =\frac{P_{t}-P_{c}}{\omega_{t c} J_{t c}}-w_{f r i c} \omega_{t c}^{2}
\end{aligned}
$$

Where $\dot{m}_{x}$ denote massflows, $T_{i m / e m}$ manifold temperatures, $J_{\text {genset } / \text { tc }}$ inertias, $V_{i m / e m}$ manifold volumes, $R_{a / e}$ gas constants, $P_{t / c}$ turbine/compressor powers, $T_{i c e}$ engine torque, and $P_{\text {mech }}$ mechanical generator power, with connections between the components as in Fig 2. For further explanation of the symbols, see Table A.1 in the appendix. There is also a summation state, to keep track of the produced energy:

$$
\frac{d E_{g e n}}{d t}=P_{g e n}
$$

The model consists of ten submodels, connected as seen in Fig. 2. The submodels are models for compressor massflow and power, intake manifold pressure, engine torque and exhaust temperature, exhaust manifold pressure, wastegate massflow, turbine massflow and power, generator losses, and engine and turbocharger speed.

\section{PROBLEM FORMULATION}

The considered problem is that the GENSET is at idle when the operator requests a step in power. Previous papers have studied how to best exploit the extra degree of

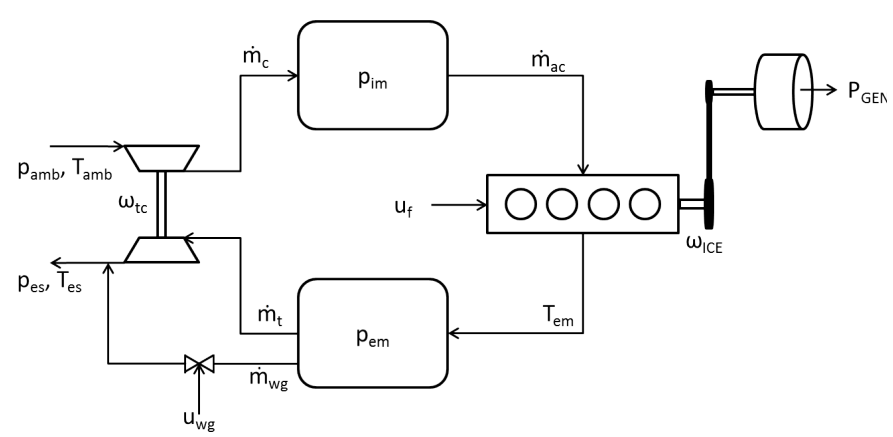

Fig. 2. Structure of the MVEM. The modeled components as well as the connection between them.

freedom available in a diesel-electric and optimally control the GENSET from idle to target power and energy by solving the two optimization problems:

$$
\begin{array}{ll}
\min _{u(t)} & \int_{0}^{T} \dot{m}_{f}(x(t), u(t)) \mathrm{dt} \quad \text { or } \quad \min _{u(t)} T \\
\text { s.t. } & \dot{x}(t)=f(x(t), u(t)) \\
& (x(t), u(t)) \in \Omega(t)
\end{array}
$$

where $x$ is the state vector of the model, $\dot{x}$ and is defined by (1)-(4) and $u=\left[u_{f}, u_{w g}, P_{g e n}\right]$. The considered problem is a step from idle to a requested output power, $P_{r e q}$, augmented with that a certain amount of energy, $E_{r e q}$ has to be produced. $E_{r e q}$ can be interpreted as a short driving mission, and also as a measure on the amount of freedom given to the powertrain, in terms of produced energy, before the operators power request has to be met. The studied transients from idle to a target power and energy are also subject to time varying constraints imposed by the components, such as maximum torque and minimum speed, and also a requirement that the control has to end in a stationary point. The time varying constraints $(x(t), u(t)) \in \Omega(t)$ are:

$$
\begin{array}{ll}
x(0)=x 0, & \dot{x}(T)=0 \\
u_{\text {min }} \leq u(t) \leq u_{\text {max }}, & x_{\text {min }} \leq x(t) \leq x_{\text {max }} \\
T_{i c e}(x(t), u(t)) \leq T_{\text {ice, } \max }(x(t)), & P_{\text {gen }}(T)=P_{\text {req }} \\
0 \leq P_{\text {gen }}(t) \leq P_{\text {req }}, & E_{\text {gen }}(T)=E_{\text {req }} \\
\phi_{\lambda}(x(t), u(t)) \geq 0 &
\end{array}
$$$$
\text { For all problems studied in this paper } P_{r e q}=170 \mathrm{~kW} \text {. }
$$

\section{OPTIMAL CONTROL TRAJECTORIES}

The resulting engine torque-engine speed trajectories to (6) for $E_{r e q}=340 \mathrm{~kJ}$ and $E_{r e q} \geq 0 \mathrm{~kJ}$, are shown in Fig. 3. Also shown is the minimum fuel solution for fixed output power, denoted $\min m_{f, 2-\text { phase. }}$ In $\min m_{f, 2-p h a s e}$ the problem is solved using two phases with the added constraints that in phase $1 P_{\text {gen }}=0$ and in phase 2 $P_{g e n}=P_{r e q}$. For a more thorough discussion on the optimal results, see Sivertsson and Eriksson [2013a,b].

With $E_{r e q} \geq 0$ the solutions for the two criteria are very similar. The optimal control puts as much energy as possible into the system, following the smoke-limiter and maximum torque line. The difference between the solutions to the two criteria is which operating point they approach and also the fine tuning to get there.

When $E_{r e q}>0$ the solutions differ. For $\min T$ and $\min m_{f_{2-p h a s e}}$ the characteristics are the same, and also 


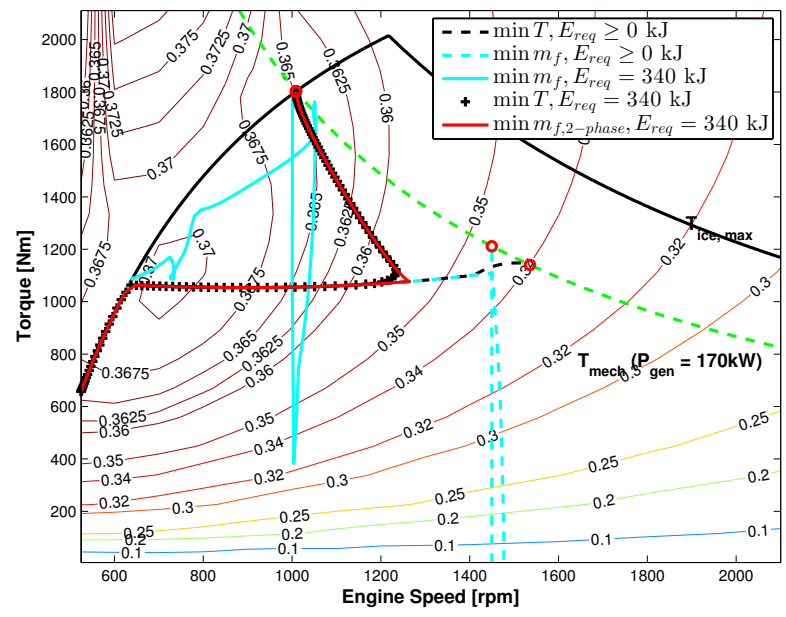

Fig. 3. The fuel and time optimal trajectories for different $E_{\text {req. }}$.

independent of $E_{r e q}$. The optimal solution is to accelerate the engine, following the smoke-limiter, and then use the excess kinetic energy to produce power and approach the maximum efficiency point for the requested power. At which engine speed this step occurs does however depend on the requested power.

For min $m_{f}$ the solution changes with $E_{r e q}$. The control is to accelerate whilst producing power and if $E_{r e q}$ is large enough, have a stationary phase at the peak efficiency of the GENSET before a final acceleration to meet the end constraints, see Fig. 3. The final operating point is then approached from a higher engine speed.

\section{OPTIMAL LINE}

In Cairano et al. [2012], Yoo et al. [2009] the change in requested power is limited to be able to maintain the GENSET close to its stationary optimal line. To study how far from optimal this strategy is for turbocharged GENSETs the problem in (6) is solved with the added constraint that the engine power, $P_{i c e}=\omega_{i c e} T_{i c e}$, is not allowed to deviate more than $1 \mathrm{~kW}$ from the stationary optimal line. As seen in Fig. 4 the optimal line is nonsmooth therefore a fifth order polynomial approximation of the optimal line, also visible in Fig. 4, is used instead. The added constraint is of the form:

$$
P_{\text {opt }}\left(\omega_{\text {ice }}\right)-1 k W \leq P_{\text {ice }} \leq P_{\text {opt }}\left(\omega_{i c e}\right)+1 k W
$$

Comparing Fig. 3-4 it is seen that the optimal solutions does not follow the optimal line neither for minimum time nor minimum fuel. Further, restricting the control to follow the optimal line the control cannot reach a point where it can sustain $P_{g e n}=170 \mathrm{~kW}$ without producing output power, since the control needs to build turbocharger speed and intake manifold pressure without accelerating the GENSET. In order to reach the final operating point the produced energy is $E_{g e n}=[305,320] \mathrm{kJ}$ for $\min T$ and $\min m_{f}$ respectively. This means that the operator or controller has to request $P_{\text {req }}=170 \mathrm{~kW}$ for $2.7-2.8 \mathrm{~s}$ for this power to be realizable, a problem the optimal control does not have. However to make the comparison fair the strategies are evaluated using $E_{r e q}=340 \mathrm{~kJ}$, and the

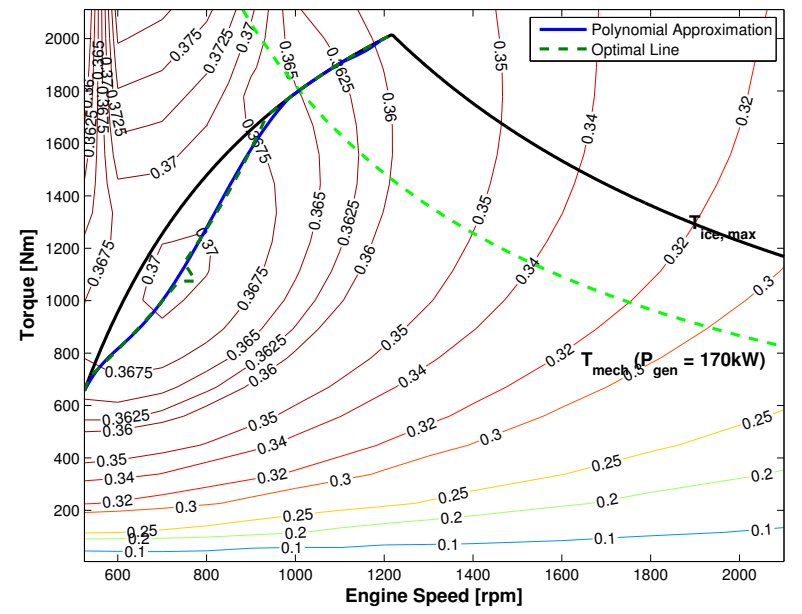

Fig. 4. The stationary optimal operating line and its polynomial approximation.

results are expressed relative the time optimal solution for $E_{r e q}=340 \mathrm{~kJ}$, shown in Table 1 . There it is seen that even though the optimal trajectories do not follow the stationary optimal line, following the optimal line gives almost optimal fuel economy, the difference is just $0.3-0.4 \%$ depending on criteria. Following the optimal line is also substantially faster than the $\min m_{f}$ solution. However, the $\min T$ solution consumes just $1.4-1.7 \%$ more fuel than the $\min m_{f}$ and optimal line strategies but is $27.7-65.2 \%$ faster.

\section{CONTROL USING SAE J1939}

In the optimization it is assumed that the actuators in the GENSET can be individually controlled, this is commonly not the case. A common approach in GENSET control is to split the control in two parts, engine and generator control. The engine is controlled using the SAE J1939standard, following either a speed or torque reference. The controller parameters are tuned first by iterating through a large set of possible candidates and then selecting the best one as initial guess for an optimization problem solved with fmincon in Matlab to fine tune the performance. In the following control strategies the wastegate is assumed fully closed throughout the transient, i.e. $u_{w g}=0$.

\subsection{Strategy 1: Engine Speed Controlled}

The normal GENSET control is that the engine tries to follow a reference speed, see Lee et al. [2008], Cooper et al. [2009], Leuchter et al. [2007]. From talks with the industry the standard generator control scheme for propulsion applications is to reduce the produced power from the desired power based on the speed error of the engine. The scheme can be summarized as:

$$
\begin{aligned}
\omega_{i c e, e r r} & =\omega_{i c e, r e f}-\omega_{i c e} \\
u_{f} & =\operatorname{sat}\left(k_{p, \omega} \omega_{i c e, e r r}+k_{i, \omega} \int_{0}^{T} \omega_{i c e, e r r} \mathrm{dt}\right) \\
P_{\text {gen }} & =\operatorname{sat}\left(P_{\text {gen }, \text { ref }}-k_{p, \text { gen }} \omega_{\text {red }}\right) \\
\omega_{\text {red }} & = \begin{cases}\omega_{\text {ice,err }}-\omega_{d} & \text { if } \omega_{\text {ice }, \text { err }}-\omega_{d} \geq 0 \\
0 & \text { otherwise }\end{cases}
\end{aligned}
$$


Where $\omega_{d}$ is a dead-zone to circumvent the drawback that the speed error has to be zero for the requested power to be produced. sat $\left(u_{x}\right)$ means that the control is saturated to comply with the constraints. For $u_{f}$ this means both smoke-limited, max torque-limited as well as being limited by the maximum possible fuel injection. Here the gains for the engine speed controller, $k_{p}$ and $k_{i}$, are tuned to correspond to the speed controller of the modeled engine. The Strategy 1 (S1) control thus has three parameters/setpoints.

\subsection{Strategy 2: Engine Load Controlled}

Instead of using the speed request of the SAE J1939standard to control the engine, one could use the load request and instead use the generator to control the speed of the GENSET. From a $P_{\text {req }}$ this strategy then requires two setpoints, desired torque and speed. From a $P_{r e q}$ and $\omega_{\text {ice,ref }}$ the mechanical torque of the generator, $T_{\text {mech }}$, is calculated. This torque is then sent to the engine control system. In simulation the torque model is inverted to calculate the fuel control. The generator power is set by a PI-controller from the engine speed error. A drawback is that $P_{\text {gen }}$ is not allowed to exceed $P_{r e q}$, since that would require a consumer being able to accept the excess power. This means that the generator cannot control the engine speed if $\omega_{i c e, e r r}<0$ since potentially $\omega_{i c e} T_{i c e}>$ $P_{\text {mech }}\left(\omega_{i c e}, P_{\text {req }}\right)$. A solution to this problem is to instead decrease the desired torque proportional to the unavailable torque desired from the generator by the controller.

The suggested strategy is then summarized as:

$$
\begin{aligned}
P_{g e n, s p} & =k_{p, P} \omega_{i c e, e r r}+k_{i, P} \int_{0}^{T} \omega_{i c e, e r r} \mathrm{dt} \\
P_{\text {gen }} & =\operatorname{sat}\left(P_{\text {gen }, s p}\right) \\
T_{\text {red }} & = \begin{cases}\frac{P_{\text {gen }, s p}-P_{\text {gen }}}{\omega_{i c e}} & \text { if } P_{\text {gen }, s p}-P_{g e n} \geq 0 \\
0 & \text { otherwise }\end{cases} \\
T_{\text {ref }} & =\frac{P_{\text {mech }}\left(P_{\text {req }}, \omega_{i c e, r e f}\right)}{\omega_{i c e, r e f}}-k_{p, T} T_{r e d} \\
u_{f} & =\operatorname{sat}\left(f\left(\omega_{\text {ice }}, T_{\text {ref }}, p_{i m}, p_{\text {em }}\right)\right)
\end{aligned}
$$

The Strategy 2 (S2) controller then has three tuning parameters, $k_{p, P}, k_{i, P}$, and $k_{p, T}$.

\subsection{Results and Discussion}

To investigate the potential for optimal control, the gains in the two different controllers are tuned for the different criteria, $P_{r e q}$ and $E_{r e q}$. The previously solved optimal control problem, (6), requires the end point to be stationary, $P_{g e n}(T)=P_{r e q}, E_{g e n}(T)=E_{r e q}$, as well as component and environmental constraints to be fulfilled. To request stationarity and that the power and energy should be met is infeasible for lower $E_{r e q}$ when using PI-controllers due to the turbocharger dynamics, since $E_{r e q}$ will be met before the target speed and stationary conditions are reached. For S1 the stationarity requirement is therefore removed, since the generator power is decreased if the speed error increases. For S2 it is replaced with the requirement $0 \leq \omega_{\text {ice } \text { err }}(T) \leq 0.52 \mathrm{rad} / \mathrm{s}$.

In Fig. 5 the resulting torque-speed trajectories for the two different controllers are shown. The gains are tuned for

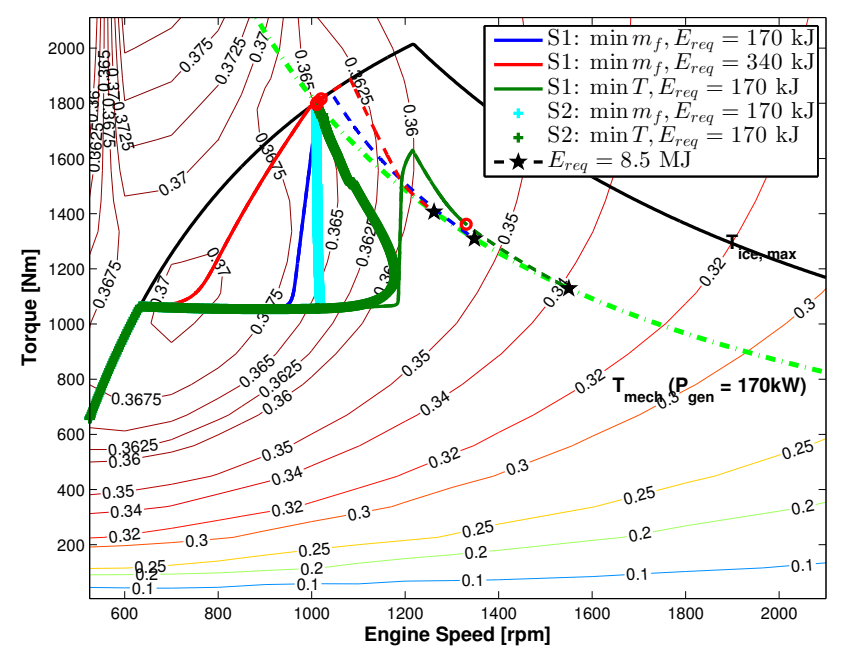

Fig. 5. The resulting engine torque-engine speed trajectories for the two control strategies, with gains optimized for $E_{r e q}=[170,340] \mathrm{kJ}$ but also simulated for $E_{r e q}=8.5$ MJ. Solid and + are optimized results with red circles marking the end points, dashed are simulated until $E_{r e q}=8.5 \mathrm{MJ}$ with pentagrams marking the end points.

$E_{r e q}=[170,340] \mathrm{kJ}$ but for S2 both $E_{\text {req }}$ have the same solution why $E_{r e q}=340 \mathrm{~kJ}$ is left out. For S1 the controller is also simulated and plotted for for $E_{r e q}=8.5 \mathrm{MJ}$ to show how $\omega_{\text {ice, ref }}$ is used.

With S1 it is not optimal to request the speed of peak efficiency, something also seen in Fig. 5 that $\omega_{i c e, r e f}$, marked by red stars, is quite far from the peak efficiency region. This since the requested engine speed cannot be met unless $E_{r e q}$ is very large and for low $\omega_{\text {ice, ref }} P_{r e q}$ cannot be met without exceeding $E_{r e q}$, since it is necessary to build turbocharger speed to be able to produce the high torques required. For $\min m_{f}$ the parameters are instead such that the GENSET stays in the high efficiency region, for $\min T$ the ability to meet $P_{r e q}$ dominates.

With $\mathrm{S} 2$ it is both fuel and time optimal to set $\omega_{\text {ice, ref }}$ in the peak efficiency region. For $\min m_{f}$ the end point is approached with very little overshoot in engine speed, whereas for $\min T$ the overshoot is larger, similar to the time optimal trajectories.

In Table 1 the fuel consumption and duration are related to the time optimal results. These controllers are not far from fuel optimal when tuned for $\min m_{f}$, and not far from time optimal when tuned for $\min T$. For S1 the punishment in the metric it is not tuned for is substantial, i.e. the fuel consumption increases with $8 \%$ when the controller is tuned for $\min T$ and the duration increases with $25 \%$ when tuned for min $m_{f}$. With S2 this is avoided with and the controller performs well in both metrics regardless for which it is tuned. However the potential fuel economy of $\mathrm{S} 1$ is higher than S2, whereas $\mathrm{S} 2$ is faster than S1. Worth noting is that $\mathrm{S} 2 \min T$ is very close to the time optimal solution in both metrics, and the trajectory is also qualitatively similar seen when comparing Fig. 4 and 5. 


\section{OPTIMAL CONTROL USING SAE J1939}

Even though the implemented strategies S1 and S2 can come close to the optimal solutions the gains of the controllers end up quite extreme, tuned for a specific criteria. The question whether or not the optimal trajectories are implementable using the SAE J1939-standard is still open. To evaluate this min $m_{f, 2-p h a s e}$ is selected since it represents a good trade-off between fuel and duration, and also since it is rather simple. First it accelerates along the smoke-limiter up to a certain engine speed, $\omega_{\text {step }}$, and then applies a step in generator power from zero to $P_{\text {req }}$, a power that is then held until the end. The wastegate is used to maintain the engine on the smoke-limit. Here the wastegate is ignored and again assumed closed throughout the transient.

\subsection{Optimal control with the engine speed controlled (S1)}

For S1 this means that first a $\omega_{i c e, r e f, 1}$ higher than $\omega_{\text {step }}$ is sent to the engine speed controller. Since $P_{\text {gen }}$ should be zero the $P_{\text {gen }}$ control has to be disconnected. When $\omega_{i c e}=\omega_{\text {step }}, P_{\text {gen }}=P_{\text {req }}$ and this power should then be maintained and if $\omega_{\text {step }}$ is correct the engine speed should decrease. When $\frac{d \omega_{i c e}}{d t} \geq 0$ the speed reference is set to $\omega_{i c e, r e f}$. For this to work the integrator in the engine speed controller needs to be reset to a value fitting the target operating point, which makes the control sensitive to errors and integral wind-up. When this shift of reference occurs the generator control can be activated since now the reference speed is the target for control, not just a value to ensure that the control follows the smoke-limiter. This control increases the number of control parameters with one, since $\omega_{i c e, r e f, 1}$ is just set to a value higher than $\omega_{\text {step }}$, which means that only $\omega_{\text {step }}$ and $\omega_{i c e, r e f}$ need to be decided.

\subsection{Optimal control with the engine Load controlled (S2)}

Using S2 the torque reference is calculated using $\omega_{\text {ice,ref }}$ and $P_{r e q}$. The difference here compared to $\mathrm{S} 2$ is that the generator is not activated by exceeding $\omega_{\text {ice,ref }}$, but by exceeding $\omega_{\text {step }}$. When this speed is exceeded $P_{\text {gen }}=$ sat $\left(P_{\text {gen }, s p}\right)$ calculated according to $(12)$ with the integrator part set to $P_{r e q}$. To avoid integral wind-up this is reset to $P_{r e q}$ when $\omega_{i c e, e r r}=0$. To avoid decreasing the reference when it is not necessary $T_{r e d}=0$ and only activated if the step has occurred and $\frac{d \omega_{\text {ice }}}{d t}>0$. When $\omega_{\text {ice,err }}=0$ it is then reset to zero. This scheme then only has one extra parameter, $\omega_{\text {step }}$.

\subsection{Results and discussion}

For both strategies $\omega_{\text {step }}$ and $\omega_{i c e, \text { ref }}$ need to be decided. $\omega_{i c e, r e f}$ can be found from stationary measurements, however $\omega_{\text {step }}$ is not as easily defined. To investigate the controllers sensitivity to error in this parameter it is varied $\omega_{\text {opt }} \pm 10 \%$ where $\omega_{\text {opt }}$ is the speed where the step occurs in the optimal $\min m_{f, 2-\text { phase }}$ solution shown in Fig. 4. $\omega_{\text {ice,ref }}$ is decided as the end operating point from that solution. The results are shown in Fig. 6 and in Table 1.

For both strategies it is possible to control the GENSET in an optimal manner, both controllers end up being as
Table 1. Change in fuel and time compared to $\min T, E_{\text {req }}=340 \mathrm{~kJ}$.

\begin{tabular}{ccc}
\hline & $\Delta m_{f}[\%]$ & $\Delta T[\%]$ \\
\hline $\min T$ & 0.0 & 0.0 \\
$\min m_{f}$ & -1.7 & 65.2 \\
$\min m_{f, 2-\text { phase }}$ & 0.1 & 0.0 \\
\hline $\min T_{\text {opt-line }}$ & -1.4 & 27.7 \\
$\min m_{f, \text { opt-line }}$ & -1.5 & 38.3 \\
\hline $\mathrm{S} 1: \min T$ & 8.0 & 0.8 \\
$\mathrm{~S} 1: \min m_{f}$ & -1.2 & 25.2 \\
$\mathrm{~S} 2: \min T$ & 0.0 & 0.3 \\
$\mathrm{~S} 2: \min m_{f}$ & -0.1 & 3.8 \\
\hline $\mathrm{S} 1:$ Opt-control & 0.2 & 0.0 \\
$\mathrm{~S} 2:$ Opt-control & 0.1 & 0.0 \\
\hline
\end{tabular}

fast and roughly as fuel efficient as the optimal solution. For $\mathrm{S} 1$ the control is however quite sensitive to errors in $\omega_{\text {step }}$. It also has the drawback that the integrator of the engine speed controller needs to be reset, something that is not available in the SAE J1939 standard. In Fig. 6 the used gains are in the same range as for $\mathrm{S} 1: \min m_{f}, E_{r e q}=$ $340 \mathrm{~kJ}$. With $10 \%$ error in $\omega_{\text {step }}$ the control ends up with the engine stalling, indicating that this control strategy is not very robust. For S2 the gains are set to reasonable values, not tuned for a specific criteria. S2 does not have the drawback of changing reference as with S1, looking at Fig. 6 is is also robust to errors in $\omega_{\text {step }}$. Despite errors of $10 \%$ the control manages to bring the GENSET to stationarity in speed and power within $1.5 \mathrm{~s}$.

\section{CONCLUSION}

In this paper the performance of several different control strategies for a diesel-electric powertrain in transient operation are discussed and evaluated compared to the optimal control trajectories. The considered problem is that the GENSET starts at idle and the operator requests a certain power, $P_{r e q}$ a power that should be met either as fast or as fuel efficient as possible. To make the controllers comparable this is augmented with that a certain amount of energy has to be produced. The controllers are then evaluated in terms of duration and fuel economy compared to the minimum fuel and minimum time solutions.

First a strategy where the control is limited to follow the stationary optimal line is evaluated. It is seen to provide almost optimal fuel economy, it however takes almost 3s to reach the requested output power, regardless of criteria.

Then two basic PI control strategies using the same structure as used in industry are studied. The engine is controlled using the SAE J1939-standard which has the options of using speed control or load control to control the engine. The gains of the PI controllers are then tuned for minimum time or minimum fuel. With the engine speed controlled, a strategy called S1, the controller is seen to give almost optimal performance in the metric for which it was tuned, for the other metric the performance is not as good. With the engine load controlled, called S2, the resulting solutions represents a better trade-off between the two metrics, while still being close the optimal results.

Finally it is shown that the optimal trajectories could be implemented using the SAE J1939-standard, both with the engine speed controlled and with the engine load 

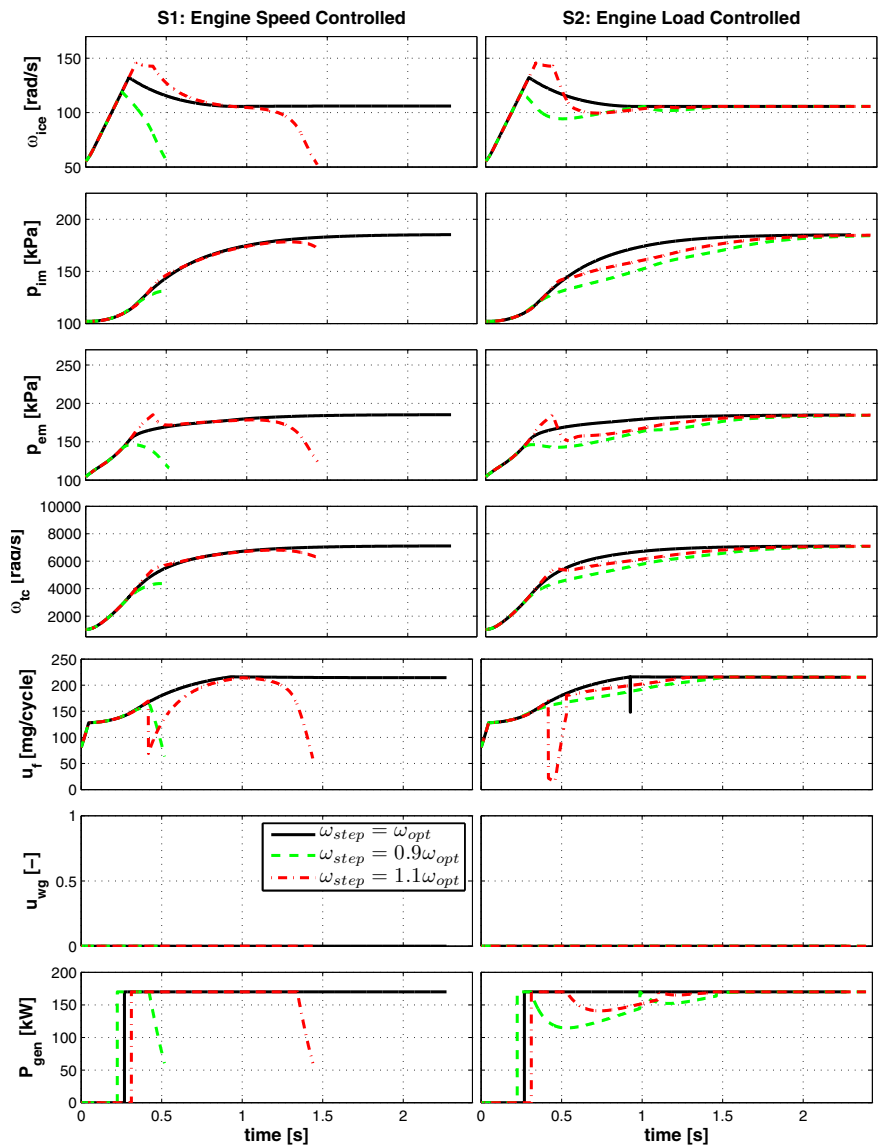

Fig. 6. Optimal control using $\mathrm{S} 1$ and $\mathrm{S} 2 . \mathrm{S} 2$ is robust to errors in $\omega_{\text {step }}, \mathrm{S} 1$ is not.

controlled. With the engine speed controlled this involves switching speed reference and resetting of the internal speed controller of the engine, something that may not be possible. It is also seen that the control is not robust to errors in one of the parameters describing the optimal solution. With the engine load controlled on the other hand the reference sent to the engine is in the ideal case constant throughout the transient and even with errors it is changed in a less dramatic way. The resulting controller is also seen to be robust to errors and to able to bring the engine speed and output power to stationarity within $1.5 \mathrm{~s}$.

\section{REFERENCES}

S Di Cairano, W Liang, I V Kolmanovsky, M L Kuang, and A M Phillips. Power smoothing energy management and its application to a series hybrid powertrain. Accepted for publication in IEEE Transactions on Control Systems Technology, 2012.

A. R. Cooper, D.J. Morrow, and K. D R Chambers. A turbocharged diesel generator set model. In Universities Power Engineering Conference (UPEC), 2009 Proceedings of the 44th International, pages 1-5, 2009.

Joon-Hwan Lee, Seung-Hwan Lee, and Seung-Ki Sul. Variable speed engine generator with super-capacitor; isolated power generation system and fuel efficiency. In Industry Applications Society Annual Meeting, 2008. IAS '08. IEEE, pages 1-5, 2008.

J. Leuchter, V. Refucha, Z. Krupka, and P. Bauer. Dynamic behavior of mobile generator set with variable speed and diesel engine. In Power Electronics Specialists Conference, 200\%. PESC 200\%. IEEE, pages 2287-2293, 2007.

Constantine D Rakopoulos and Evangelos G Giakoumis. Diesel engine transient operation - Principles of operation and simulation analysis. Springer, 2009.

SAE J1939 Standard. "http://standards.sae.org/ j1939/71_201002/", 2013. Read 2013-10-04.

V Sezer, M Gokasan, and S Bogosyan. A novel ecms and combined cost map approach for high-efficiency series hybrid electric vehicles. IEEE Transactions on Vehicular Technology, 60(8):3557-3570, 2011.

Martin Sivertsson and Lars Eriksson. Time and fuel optimal power response of a diesel-electric powertrain. In E-COSM'12 - IFAC Workshop on Engine and Powertrain Control, Simulation and Modeling, Paris, France, October 2012.

Martin Sivertsson and Lars Eriksson. Optimal transient control and effects of a small energy storage for a diesel-electric powertrain. In $A A C^{\prime} 13-T h e$ 7th IFAC Symposium on Advances in Automotive Control, Tokyo, Japan, September 2013a.

Martin Sivertsson and Lars Eriksson. Generator effects on the optimal control of a power assisted diesel-electric powertrain. In IEEE VPPC 2013 - The 9th IEEE Vehicle Power and Propulsion Conference, Beijing, China, October 2013b.

Hyunjae Yoo, Byung-Geuk Cho, Seung-Ki Sul, Sang-Min Kim, and Yongho Park. A power flow control strategy for optimal fuel efficiency of a variable speed enginegenerator based series hybrid electric vehicle. In IEEE ECCE'09 Energy Conversion Congress and Exposition, 2009.

Appendix A

Table A.1. Model nomenclature

\begin{tabular}{cc}
\hline \hline Symbol & Description \\
\hline$\omega_{i c e}$ & Engine Speed \\
$p_{i m}$ & Intake manifold pressure \\
$p_{e m}$ & Exhaust manifold pressure \\
$\omega_{t c}$ & Turbocharger speed \\
$u_{f}$ & Injected fuel per cycle \\
$u_{w g}$ & Wastegate position \\
$P_{g e n}$ & Electrical generator power \\
$P_{m e c h}$ & Mechanical generator power \\
$T_{i c e}$ & Engine torque \\
$\dot{m}_{c}$ & Compressor massflow \\
$\dot{m}_{a c}$ & Air massflow into the cylinders \\
$\dot{m}_{f}$ & Fuel massflow \\
$\dot{m}_{t}$ & Turbine massflow \\
$\dot{m}_{w g}$ & Wastegate massflow \\
$T_{e m}$ & Exhaust manifold temperature \\
$P_{c}$ & Compressor power \\
$P_{t}$ & Turbine power \\
\hline$J_{g e n s e t}$ & GENSET inertia \\
$J_{t c}$ & Turbocharger inertia \\
$T_{i m}$ & Intake manifold temperature \\
$R_{a / e}$ & Gas constant air/exhaust gas \\
$V_{i s}$ & Volume of intake system \\
$V_{e m}$ & Volume of exhaust manifold \\
$w_{f r i c}$ & Friction coefficient, turbocharger \\
\hline &
\end{tabular}

